\title{
The Brugada Syndrome. Outcome of one Case
}

\author{
Ivan G. Maia, Magda Wanderley Soares, Silvia H. Boghossian, Roberto Sá
}

Rio de Janeiro, RJ - Brazil

\begin{abstract}
The Brugada syndrome is a rare condition, and due to its mutating manner of presentation it may be difficult to diagnose. We report one case and discuss the diagnostic aspects and the clinical outcome of one patient with characteristic findings of this syndrome. These findings are especially defined by J-ST elevation in the right leads of serial electrocardiographic records, wide oscillations of J points and ST segments during 24-hour Holter monitoring, and nocturnal sudden death. We stress the importance of the Holter monitor findings for diagnostic complementation. Through this method it is possible to establish a correlation between vigil activities and sleep and the variability of the degree of impairment in ventricular repolarization.
\end{abstract}

In the last decade, interest in the study of the primary electrical diseases of the myocardium has progressively risen. The conjunct effort of specialists in arrhythmias, electrophysiologists, and geneticists has allowed clarification of several pathophysiological and clinical characteristics of those diseases with a favorable influence on their prognosis. Among those that have been drawing more attention, we can cite the congenital long QT syndrome and, more recently, the Brugada syndrome $\mathrm{e}^{1,2}$. Both have as a common characteristic, when evolving in their natural form, the presence of a high potential for developing severe ventricular arrhythmias producing syncopal findings, sudden death, or both. Therefore, taking this prognosis and an often times dubious diagnosis into consideration, publishing data related to the evolution of a patient with the Brugada syndrome seemed worthwhile.

\section{Case Report}

The patient was a 40-year-old white male, from the State of Rio de Janeiro, where he had always lived. He had a history of hypertension for 5 years and use of captopril $(25 \mathrm{mg}$ / day) and hydrochlorothiazide ( $50 \mathrm{mg} /$ day).

The patient reported that in July 1998 he had hematuria and lumbar pain. Due to the presence of renal calculi, surgery (left pyelolithotomy) was indicated.

Hospital Pró-Cardíaco - Hospital de Cardiologia de Laranjeiras

Mailing address: Ivan G. Maia - Rua Raul Kennedy, 81 - 22631-200 -

Rio de Janeiro, RJ, Brazil
In the preoperative assessment, the patient was asymptomatic, smoked 40 cigarettes per day and did not consume alcohol. Prior personal and familial morbidity was not notable.

On clinical examination, the patient was eupneic, in regular condition, without any alteration on inspection. His radial pulse was regular and $66 \mathrm{bpm}$; his blood pressure was 140/80mmHg.

Complementary tests, all of which were normal, were as follows: coagulation tests, hemogram, urea, glucose, electrolytes, and urinary sediment. Transthoracic echocardiogram showed that the myocardial and valvular structures and functions were normal. Chest $\mathrm{x}$-rays showed no abnormalities. Electrocardiogram showed a pattern of complete right bundle-branch block, normal QT intervals (mean of $430 \mathrm{~ms}$ ), and a degree of dispersion of ventricular repolarization of $40 \mathrm{~ms}$, similar to that observed in healthy individuals (fig. 1).

The patient sought our hospital for assessment of the surgical risk. After a new electrocardiogram, he was advised to postpone the surgery until a complete clarification of the diagnosis could be made. The electrocardiogram record was similar to the previous one (fig. 1). During this period, the patient's preoperative assessment was completed in the hospital of origin and the patient underwent surgery to remove the renal calculus under general anesthesia. The postoperative period was uneventful, and the patient was discharged 72 hours after the procedure.

As the patient had not returned to our hospital, we called him. At the beginning of November 98 , he returned and a new electrocardiogram was performed, the results of which were similar to those of the previous ones. Twenty-four-hour Holter monitoring was also performed. Forty-eight hours after the recording, at dawn, while the patient was lying down, he had a sudden stertorous breath, a convulsive crisis, and ended up dying without medical assistance. His mother and sister were with him at the time of sudden death.

The result of the Holter monitoring was as follows: sinus rhythm, atrioventricular conduction 1:1, and normal QT intervals. Ventricular repolarization showed wide oscillations of the J points and ST segments during the 24 hours, and also in the superior channel of the recording (V1). The incidence of active arrhythmias was negligible (11 ventricular extrasystoles and 2 atrial extrasystoles during the 24 hours). The patient remained asymptomatic during the recording.

Figure 2 shows 4 significant moments in the Holter recordings, where an expressive variation in the degree of 


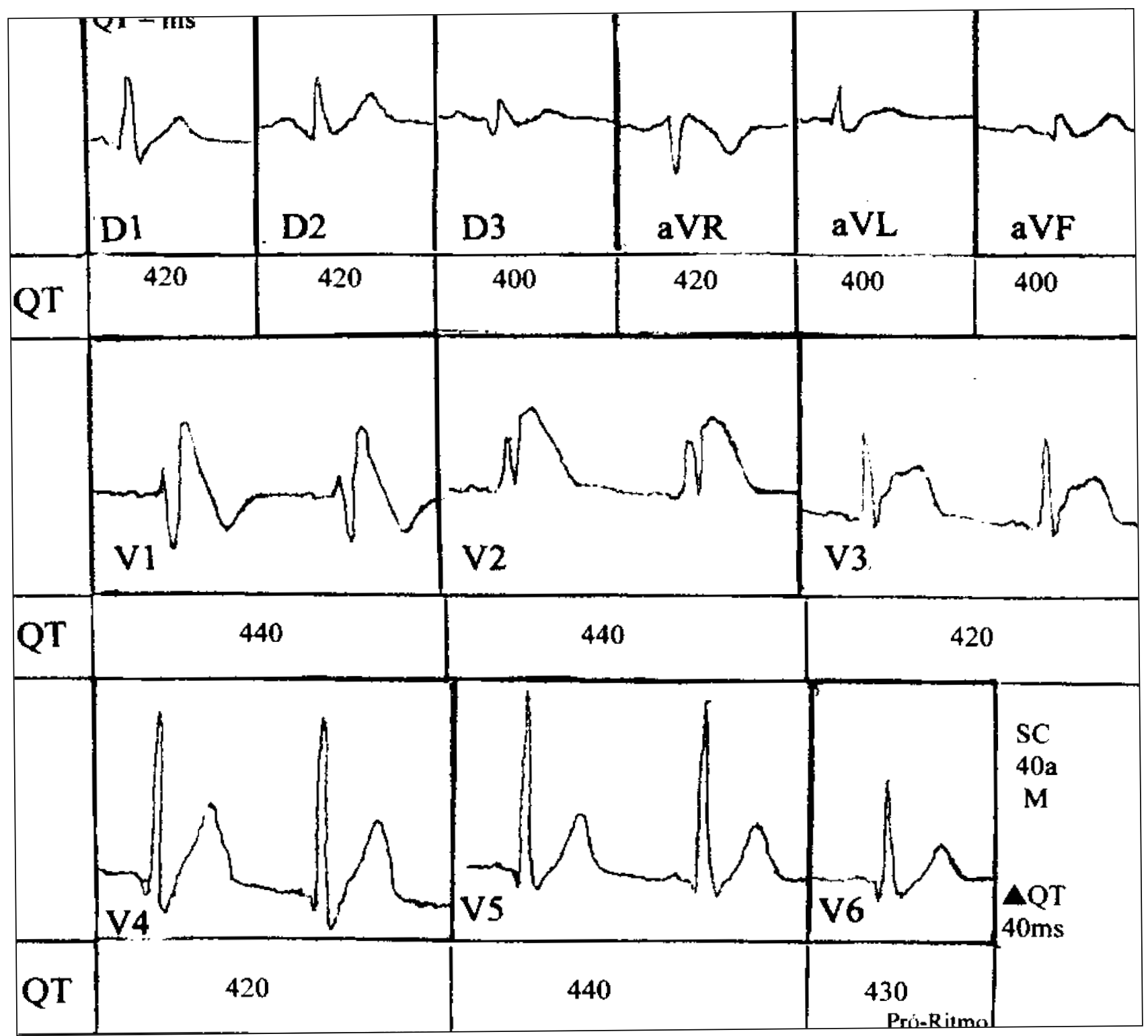

Fig. 1 -Electrocardiographic pattern of the patient. Significant alterations of the ST segment in the right precordial leads and an activation pattern of the right bundle-branch block type are notable.

elevation of the J point and ST segment can be seen, as well as its disappearance in the recordings in $\mathrm{D}$ (rapid walk).

We correlated the degree of elevation of the J point (in $\mathrm{mVolt}$ ), starting at the base line and in the $\mathrm{V} 1$ lead, with duration of the previous cardiac cycle (in ms). We determined a total of 35 cycles ranging from 460 to $1040 \mathrm{~ms}$ (the 2 extremes of frequency observed in the recordings). We found an excellent linear correlation between the 2 variables with an $\mathrm{r}=0.697(\mathrm{p}=0.001)$. Longer cardiac cycles produced a greater degree of elevation of the J point and vice-versa (fig. 3 ).

As the syndrome is genetically transmitted, we carried out a familial screening of the patient. Data were obtained through electrocardiographic findings with no therapeutical test. No episode of sudden death had occurred in the patient's family. One of his brothers had Wolff-ParkinsonWhite syndrome, a condition that is also described as of possible genetic transmission. A nephew had a mild delay in the right branch conduction, which may represent a normal variation of the electrocardiogram.

\section{Discussion}

In 1992, Brugada and Brugada ${ }^{1}$ reported a new clinical syndrome characterized by the presence of an electrocardiographic pattern similar to a right bundle-branch block associated with an elevation of the J point and ST segment (superior concavity), in the V1, V2, and V3 leads. It occurs mainly in men, who have a structurally normal heart but who may experience frequent episodes of syncope, or sudden death, or both. The condition is genetically transmitted with a dominant autosomal pattern. Elevation of the J point and ST segment is related to alterations in the dynamics of the transient current of potassium $\left(\mathrm{I}_{\mathrm{t}}\right)$, which occurs at the end of the phase 0 and beginning of the phase 2 (phase 1) 


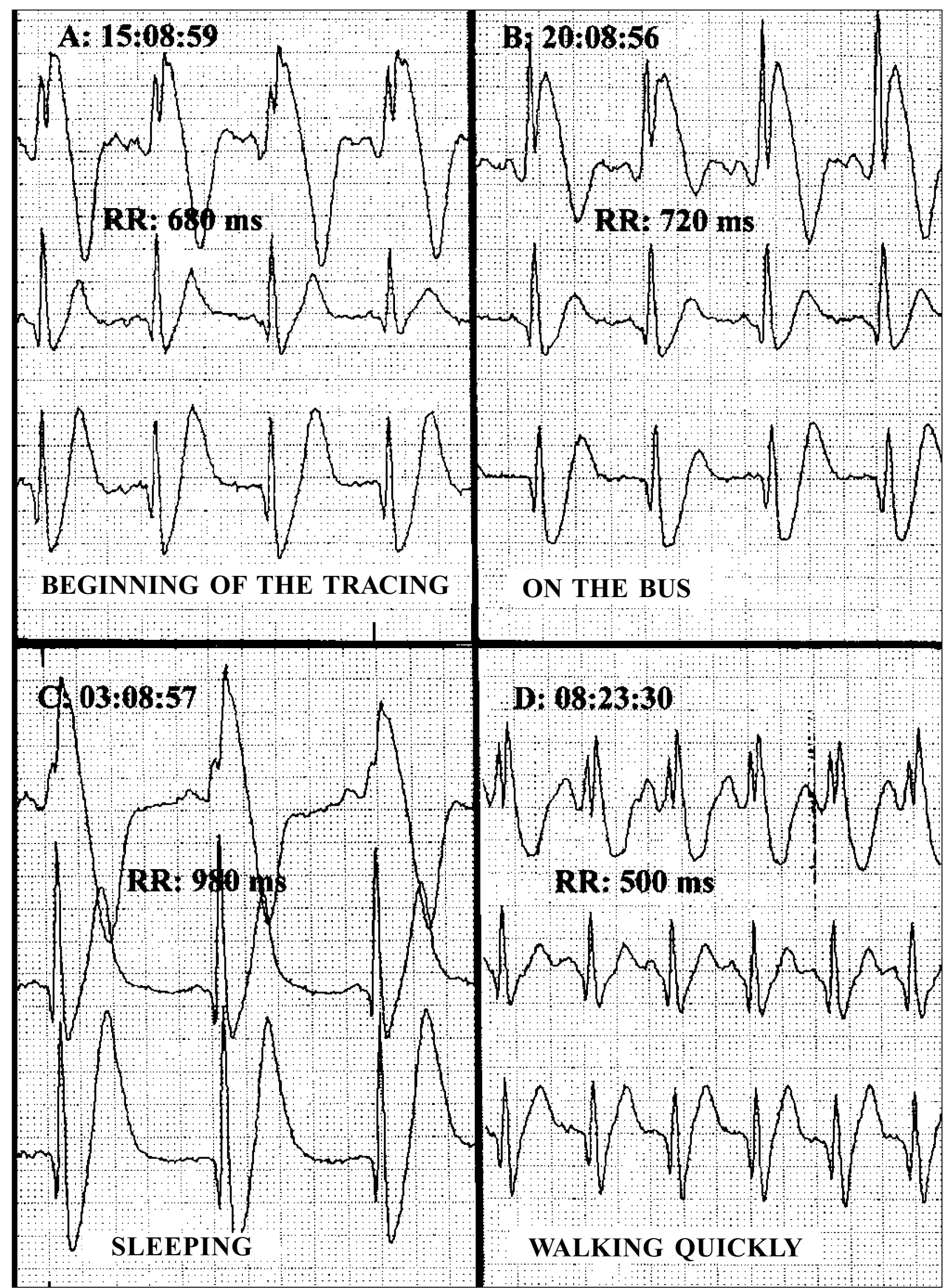

Fig. 2 - Holter monitoring recordings where the great oscillations in the degree of J-ST elevation, such as during sleep, occur (C). By walking rapidly, these alterations practically disappeared (D). In the superior channel, the V1 lead, and in the medium and inferior channels, the V5 lead. 


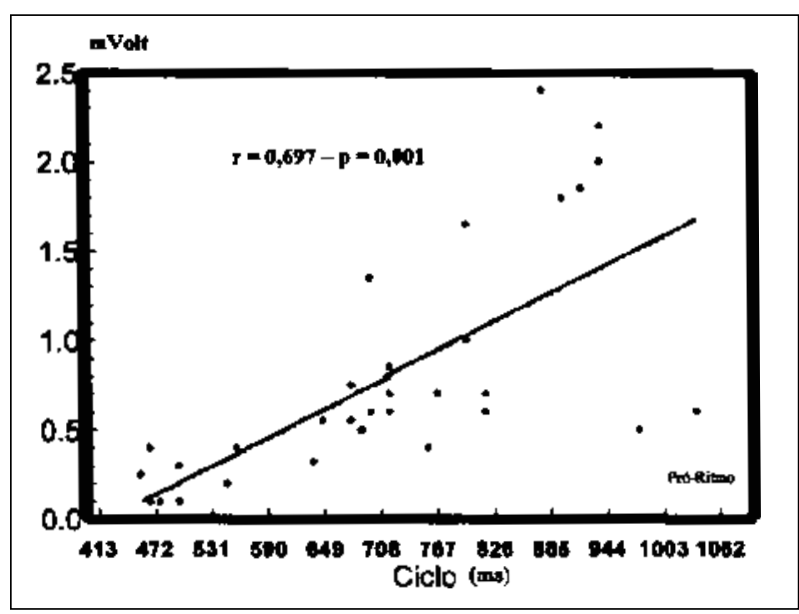

Fig. 3 - Curve of linear correlation between the degree of the J point elevation (mVolt) and duration of the previous RR intervals (ms).

of the transmembrane action potential ${ }^{2}$. The syndrome has a high incidence in some regions of the Asian continent, such as Laos and Thailand, where it accounts for most of the sudden deaths in individuals under the age of 30 years (it is estimated to cause 10 sudden deaths per 10,000 inhabitants per year) ${ }^{3}$. Even though the syndrome has already been described in North ${ }^{3}$ and South ${ }^{4}$ America and Europe ${ }^{1,2,5}$, its actual incidence in those regions is unknown.

The initial diagnostic suspicion occurs because of an electrocardiographic recording with the pattern shown in figure 1, with gross alterations of ventricular repolarization in right precordial leads. This abnormal pattern, even though possible, is extremely rare in classical right bundle-branch block ${ }^{6}$, and has never been described as a form of manifestation of an electrolytic disorder (the patient used diuretics), raising the possibility of a Brugada syndrome. As the J-ST alterations observed in this syndrome are mutant, where occult forms may occur with an apparently normal electrocardiogram (the diagnosis may only be established through a pharmacological test using ajmaline or procainamide) $)^{2,3}$, Holter monitoring has become fundamental for diagnostic confirmation. The relations between the cycle and the J-ST elevation that we observed confirm what has already been described, i. e., the exercise test tends to normalize the repo- larization alterations in this syndrome ${ }^{3}$. Considering that the presence of the J-ST elevation represents a signal of increased myocardial vulnerability, our findings explain, at least partially, the frequent occurrence of nocturnal sudden death in this syndrome, suggesting a vagal modulation for it. The nocturnal death is so frequent in certain regions of Thailand that the natives attribute it to ghosts of widows that take young males during the night. In an attempt to avoid it, young males sleep dressed in female clothes ${ }^{3}$.

This seems to be the second case of the Brugada syndrome entirely published in Brazil ${ }^{4}$. Its diagnosis was based on electrocardiographic findings, with a characteristic J-ST elevation in right precordial leads and a so-called pattern of right bundle-branch block associated with data obtained through Holter monitoring and the final clinical outcome (sudden death). From our viewpoint, these findings were enough to confirm the diagnosis of the Brugada syndrome. Unfortunately, we were not able to deepen the studies with electrophysiologic evaluations and attempt to induce ventricular tachyarrhythmias through programmed ventricular electrical stimulation.

The case is reported here to highlight the obscure prognosis of the condition. At the beginning we did not give the appropriate importance to the syndrome, and we have learned that a precocious intervention is fundamental. Even among asymptomatic patients, it is estimated that at least $1 / 3$ of them will develop ventricular tachycardia or ventricular fibrillation within the 24 months following the initial diagnosis ${ }^{3}$. This initial diagnosis is based on electrocardiographic findings, data from Holter monitoring, and on the patient's report about previous syncopes or a familial history of sudden death. Those cases of occult manifestation are of difficult diagnosis. In case of suspicion, the provocative pharmacological test with ajmaline, considered a specific drug ${ }^{3}$, is very useful. Other drugs, such as procainamide, may induce a similar response $^{7}$. The control of ventricular tachyarrhythmias with the use of antiarrhythmic drugs has not been a feasible option. Implantation of a cardioverter/defibrillator seems to be the most appropriate management, because it modifies in a very favorable way the natural history of this severe and curious syndrome ${ }^{8}$.

\section{References}

1. Brugada P, Brugada J. Right bundle branch block, persistent ST segment elevation and sudden cardiac death: a distinct clinical and electrocardiographic syndrome. J Am Coll Cardiol 1992; 20: 1391-6.

2. Gussak I, Antzelevitch C, Bjerregaard P, et al. The Brugada syndrome: clinical, electrophysiologic and genetic aspects. J Am Coll Cardiol 1999; 33: 5-11.

3. Antzelevitch C, Brugada P, Brugada J, et al. The Brugada syndrome. In: Camm AJ. Ed. Clinical Approaches to Tachyarrhythmias (vol 10). Armonk: Futura, 1999.

4. Villacorta H, Torres RAF, Castro IRS, et al. Morte súbita em paciente com bloqueio de ramo direito e elevação persistente de segmento ST. Arq Bras Cardiol 1996; 66: 229-31.

5. Lorga Filho A, Brugada P. Bloqueio de ramo direito, elevação de segmento ST de
V1 a V3 e morte súbita. O que sabemos sobre esta peculiar síndrome clínico-eletrocardiográfica. Arq Bras Cardiol 1997; 68: 205-8.

6. Tohyou JA, Nakazawa K, Ozawa A, et al. A survey in the incidence of right bundle branch block with ST segment elevation among normal population. Jpn J Electrocardiol 1995; 15: 223-6.

7. Fujuki A, Usui M, Nagasawa H, et al. ST segment elevation in the right precordial leads induced with class IC antiarrhythmic drugs: insight into the mechanisms of Brugada syndrome. J Cardiovasc Electrophysiol 1999; 10: 214-8.

8. Brugada J, Brugada R. Right bundle-branch block and ST-segment elevation in leads V1 through V3. A marker for sudden death in patients without demonstrable strutural heart disease. Circulation 1998; 97: 457-60. 